\title{
System design for Simultaneous Data Acquisition from Patient Monitor and Syringe Pumps in Intensive Care Unit
}

\author{
N. Sprunk \\ TUM Graduate School of \\ Information Science in Health (GSISH) \\ TUM Technische Universität München \\ Munich, Germany \\ Nicole.Sprunk@tum.de \\ R. Bauernschmitt \\ Klinik für Kardiologie und \\ internistische Intensivmedizin \\ Isar Herz Zentrum \\ Munich, Germany
}

\author{
M. Kaur \\ Medizinischen Fakultät LMU \\ Ludwig-Maximilians-Universität München \\ Munich, Germany
}

Abstract - The automation of tasks in an intensive care unit can be beneficial for the patient's recovery and also can reduce the workload of the physicians. That is why research is looking for different tasks, which can be performed by automated machines. One specific project, our group is working on, is the automation of blood pressure regulation with drugs for patients task of the physicians, knowledge has to be gathered about, how the physicians decide on according treatment actions. As this is an everyday task, the easiest way to analyze and understand the treatment decisions is to look at taken decisions in actual patients. For this purpose a system has been set up, which can be connected to both the patient monitor and the syringe pumps, which infuse the different drugs. There were different requirements from the technical, medical point of view and also from an ethic committee, which had to be taken into account. Given those requirements hardware was chosen and suitable software was implemented. First tests showed, that the system can be operated in the intensive care unit and provide data for further research.

Keywords: Data-mining in intensive care unit, medical devices

\section{INTRODUCTION}

Automation in medicine is in the focus of research since many years as it helps to reduce workload and hopefully costs. In our own department at the German Heart Centre we have been researching e.g. on automated hemodynamic regulation with drugs [1] or the automation of a heart-lung machine [2]. Other research groups have been working on automatic anesthesia [3] or even beds, which can turn comatose patients automatically [4] for example.

\author{
A. Mendoza G., A. Knoll \\ Institut für Informatik VI - \\ Robotics and Embedded Systems \\ TUM Technische Universität München \\ Munich, Germany
}

When developing a system, which is supposed to automatically assist a task of the medical personnel, close collaboration with physicians is necessary to understand their treatment approaches. In the early stage of developing an automated system, in this case the automated hemodynamic regulation with drugs, we let our physicians define several rules, which our control algorithms were able to work with. This first rule base consisted of 81 rules. To refine the control mechanism in this case, it would be beneficial to define even more rules. Unfortunately the manual definition and labeling of different situations is quite time-consuming and tedious work to do. That is why the data collection device, described in this publication, has been developed. It is able to record the physicians' decisions directly in the intensive care unit, where they have to take the exact same decisions every day in various patients. The collected data can then e used to analyze reactions of different patients to different drug dosages and to incorporate the taken treatment decisions into the existing control mechanism.

\section{REQUIREMENTS}

There are two main requirement lists to consider. On the one hand the chosen hardware should meet all the requirements from the technical point of view. On the other hand also some conditions were defined by the medical personnel and an ethic committee, so they could accept a data collection system in their work environment and around real patients.

Technical prerequisites are:

- $\quad$ System should be able to run at least 10 hours without restart to ensure a data interval long enough to analyze 
properly:

This is influenced by the battery life and the amount of memory disk space to save data on

- System needs to communicate with both the patient monitor and the syringe pump base station:

The communication can only be done via a RS232 port with both machines, so two RS232 ports need to be available

- System needs to operate in real-time to collect data almost continuously

- System needs to fit the hygienic conditions

The prerequisites defined by the medical personnel and the ethic committee are:

- System must not disturb the work flow in the intensive care unit in any way:

Its size should be small, so it can fit somewhere behind the usual devices and it should not produce any more alarm sounds

- System should not be connected to the main power supply, so that the patient is safe from any high voltage:

This implies that is has to be run with a battery.

- Patient data has to be treated anonymously:

No data should be saved, which makes it possible to identify a single patient

Finally it is desirable to have an easy setup of the system, so the medical personnel and not only the technicians are able to start the data collection.

\section{DATA}

The collected data includes both the signal values from the patient monitor and also the syringe pumps. The data from the patient monitor comprises of signals like mean arterial pressure (MAP), central venous pressure (CVP) or heart rate (HR). The data collected from the syringe pumps is the current speed each syringe pump is running with. The syringe pump does not send any information about the drug type or its concentration. This needs to be recorded manually. Also the age, height and weight of the patient or any preconditions or performed operations have to be saved manually to be able to take this data into account in data analysis.

\section{REALISATION}

First hardware was chosen and then the according software was implemented for this platform.

\section{A. Hardware}

Depending on the described requirements a hardware device was chosen, which had a small size and can run at least 5 hours on battery power. The beagle board provided by Special Computing (USA) was chosen as a prototype. Both the beagleboard version $\mathrm{C} 4$ and $\mathrm{C} 5$ were tested and are feasible.
The advantage of this board is that it has a full operating system available, which is saved on a SD card. When using an ubuntu system, several basic programs, already in use in our department can be reused for this specific purpose, e.g. the serial port communication. Though the beagleboard does not provide two RS232 ports for the communication with both the patient monitor and the syringe pumps simultaneously, this was solved by using an USB-to-two-RS232 converter. One USB port is available on the beagleboard. Furthermore the beagleboard has two buttons available, of which one is a restart button, which triggers the system to restart and the other button is free for a programmed user input. This button was programmed to start and stop the data logging.

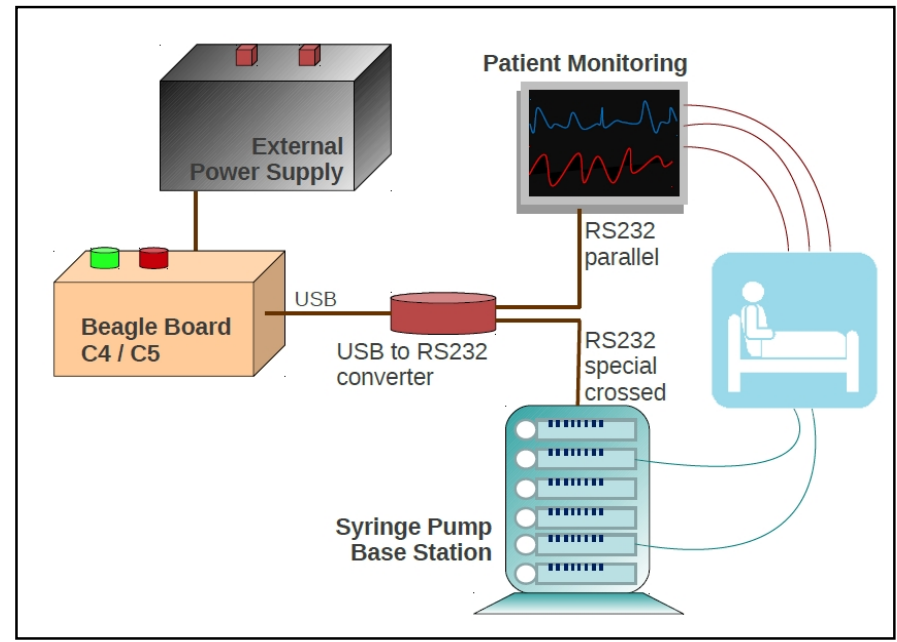

Figure 1. Hardware setup in the intensive care unit: Patient monitor and Syringe pumps are setup at the patient's side. Via according RS232 cables and an USB-to-RS232-converter the beagleboard is connected to the present machines. It is powered with an external battery pack. The beagleboard include two user input buttons, a red one for rebooting, the green one, programmed to start and stop the data collection.

As power supply an external battery is required. The beagleboard needs a 5V input. Energizer (USA) offers rechargeable power packs Energi To Go, which also have an USB output port, which can be used to power the beagleboard. The power pack XP18000, the one with the greatest capacity, was chosen to achieve the longest data collection period possible. With this power supply the data collection device can run at least 12 hours, even more depending on the frequency of data collection (default data collection frequency is every 15 seconds). Figure 1 shows the setup up of the hardware in the intensive care unit.

In the intensive care unit of our hospital, the Solar8000i of General Electrics Healthcare (USA) is used as patient monitor and the syringe pumps and their according base station are of Fresenius Kabi AG (Germany), namely Orchestra Base A with Orchestra Module DPS as syringe pump and Orchestra Module MVP as volumetric infusion pump. The connecting cable to the patient monitor Solar8000i is a standard parallel RS232 serial cable. The communication with the syringe pumps is done via the base station. The cable for this connection needs a special crossed wiring, which was soldered according to the description in the Fresenius manual description for Module DPS products. 
In figure 2 a picture of the final hardware is shown, including the beagleboard with a 8 GB SD card, the external power pack and the USB-to-Two-RS232 converter.

The USB-to-Two-RS232 converter cannot be connected directly. Due to certain prerequisites of the beagleboard it does not mount the converter ports, if it is at the only available USB

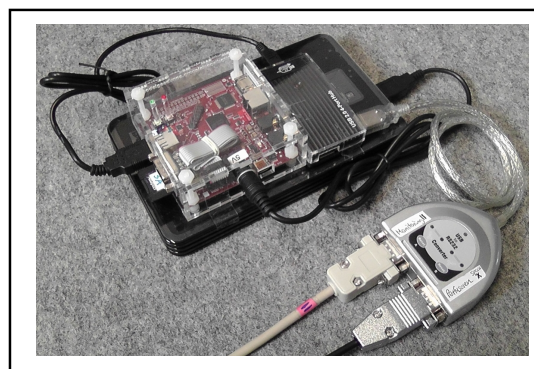

port. That is why a USB hub was used in between the USB-toTwo-RS232 converter and the beagleboard.

Figure 2. Final hardware setup: Beagleboard C5 by Special Computing (USA) with Power pack Energi To Go XP18000 of Energizer, connected via a USB-to-RS232-converter with cables to Solar8000i patient monitor of General Electrics Healthcare and the Orchestra Base A syringe pump base station of Fresenius Kabi AG (Germany)

For hygienic and safety purposes the whole hardware is sealed in a disposable sterile bag.

\section{B. Software}

With the beagleboard it is possible to have a whole ubuntu operating system with all the programming facilities, like shell script, Perl script or $\mathrm{C}++$, which were all used in the setup. Within the ubuntu system some changes were necessary to be able to have access to the user button and automatically start the according user button state script:

For the user button a file needs to be created in letc/udev/rules.d/userbutton.rules so that the user button event can be accessed via/dev/userbutton. To be able to start the data collection without actually logging in, the according script has to be started automatically. This is necessary as the final hardware device will not have a keyboard, mouse or monitor and it will not be possible to initiate a login. The only input will be the on-board user button. That is why a watchdog script is started in the end of $r c$.local. After start up this will start the PERL watchdog script in the background. The nohup will enable the command to run even after log out and furthermore produces a log script which is extended with a date/time stamp and really helps in error analysis during development. A sleep is done before the execution of the script to be sure that all components, like e.g. the ttyUSB* ports have been mounted and are available.

The watchdog script itself keeps track of any changes in the user button. If the user button is pressed, then the internal state in the watchdog script is toggled. Initially it starts off with the state "not logging", where any running logging scripts have to be stopped. Then if the user button is pressed the state "logging" is activated. If the watchdog is in the state "logging", then it checks frequently if the start_logging.pl script is running, if not it is (re)started. The start_logging.pl script keeps running until the state is changed, as this script has a further watchdog facility within.

On the other hand, if the state is "not logging", the stop_logging.pl script is called if not running yet. But it is not restarted once it finished, as it does not need to keep track of any other running processes. The purpose of the stop_logging.pl is to perform a planned shutdown of the communications. This is especially important for the communication with the Fresenius base station, because if a connection is lost, the base station will produce several alarm sounds. As one of the requirements from the medical personnel was not to have any more alarms this is avoided by telling the system to shut down the connection first.

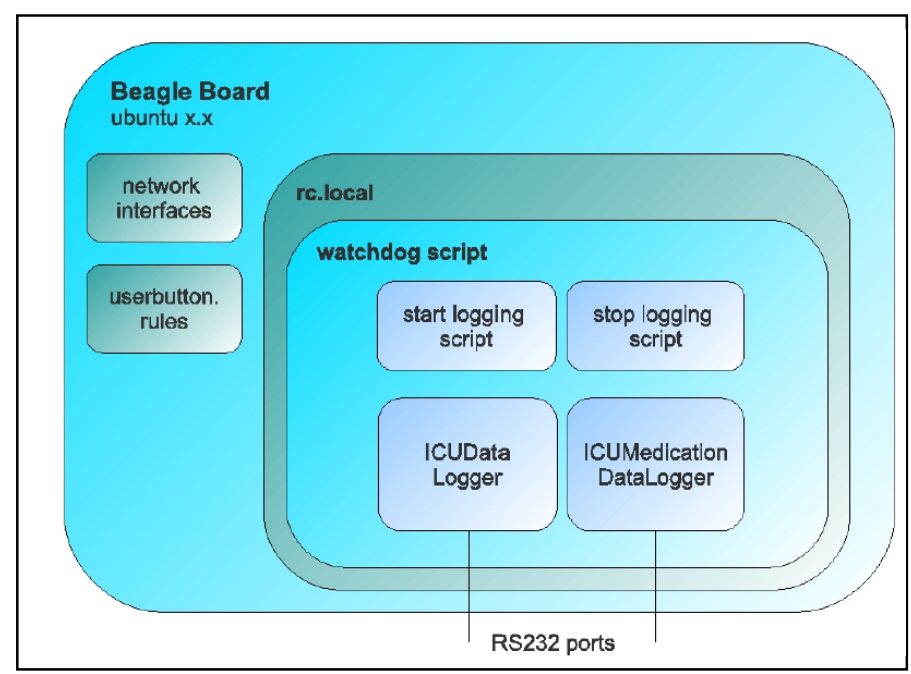

Figure 3. Software architecture on Beagle Board

The actual communication and data collection is done in two C++ executables. The two hardware devices Solar8000i by General Electrics and the base station of the Fresenius syringe pumps have specific protocols for their RS232 interface. E.g. the Fresenius base station expects an answer to its "still alive" pings, otherwise the connection is shut down. These specific protocol requirements are implemented in the ICUDataLogger and ICUMedicationDataLogger. Both communication protocols are request based, i.e. apart from "still alive" messages, data is only sent, if it was requested via serial port. It is possible to get data every 3 seconds for the Solar8000i. The Fresenius protocol does not indicate a time limit. But as we are more interested in the long term changes, the collection frequency is set to 15 seconds. This will allow observing the drug changes quite immediately but not stressing the power supply too much. The interval is sufficient for our purposes to analyze patients' reactions to dosage changes, as the pharmacodynamics and pharmacokinetics follow a relatively slow process. The drug takes time to be spread through the cardiovascular system and so does the following metabolisation, which causes the reaction.

Figure 3 shows the whole hierarchy in the software architecture of the data collection. 


\section{PATIENT ANONYMITY}

The ethic committee emphasized that the patient's privacy has to be respected. Actually the data collected via the RS232 interfaces does not provide any information about the patient, like name, date of birth or address of residency. To be able to analyze the data afterwards some information has to be collected manually, e.g. weight and height of the patient and also age and preconditions will be necessary for analysis. steep spikes (e.g. at about 22:30 and 2:00 o'clock). Probably this happens because of patient or equipment movements, but without actually knowing what exactly happened those cannot be used for analysis. Furthermore the connection with some syringe pumps sometimes fails. It is not possible to distinguish between the difference of a turned off syringe pump and a communication failure. That is why some drops in dosage changes have to be ignored manually as well. For example in

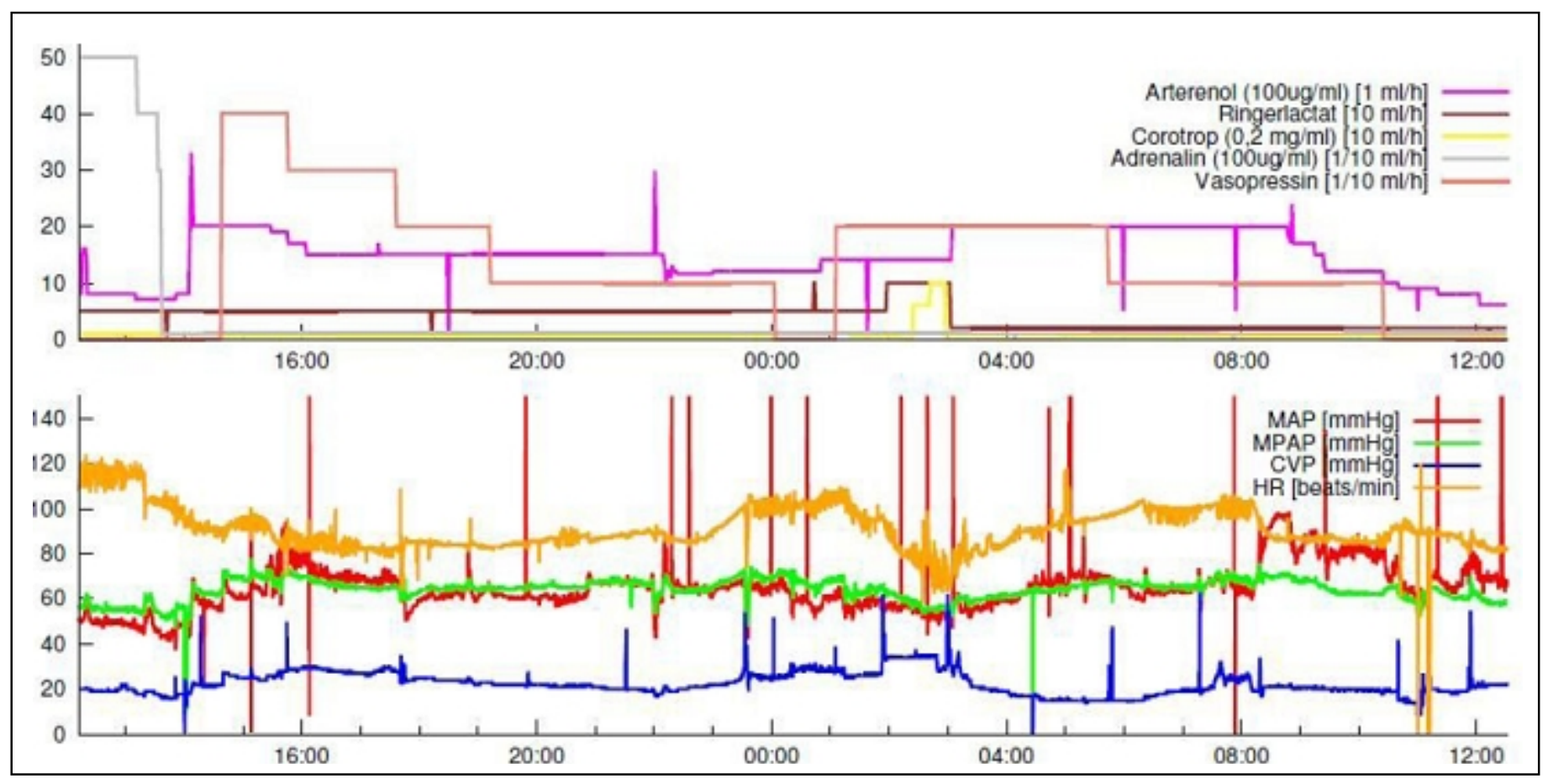

Figure 4. Data collected in a 12 hour run in the intensive care unit from patient monitor and syringe pumps

To ensure, that it will still not be possible to link a data set with a specific patient the date of the data collection is randomized.

\section{PRELIMINARY RESULTS}

After initial tests in a safe environment, the system was tested in the intensive care unit of our hospital. It collected data from intensive care patients connected to the patient monitor and the syringe pumps.

We implemented a script to create images from the collected data, which are simply several columns of numbers. Figure 4 shows the signals of a 12 hours test run in the intensive care unit. From the patient monitor only MAP, CVP, HR and MPAP (Mean pulmonary arterial pressure) are shown, although all the signals, which are connected in the patient monitor in the intensive care unit are retrieved and saved. In the syringe pumps there were five different drugs active during the time of data collection. Every time the dosages are changed, the signals and the actual change of dosage can be analyzed to better understand the decision making process to incorporate this knowledge into an initial control algorithm. However communication and signal failures have to be factored out still. For example in the MAP there occur some the diagram of Arterenol there is a drop to 0 at about 18:10 o'clock. As the dosage before and after is exactly the same, it is likely that a communication failure occurred. Overall the data looks promising to help in analysis of decision making and patient reactions to changes in drug dosages.

\section{CONCLUSION AND OUTLOOK}

A small hardware system has been setup to collect data in the intensive care unit. All requirements from the technical, medical and ethical side were met. At the moment the communication with General Electric's Solar8000i patient monitor and with Fresenius syringe pumps is implemented, but also protocols to other monitors or syringe pumps can be realized. The system ensures the safety of the patient in the intensive care unit and respects the work flow of the medical personnel, which makes regular data collections possible. These will provide us with an amount of data, which can be used for different purposes. As described before the procedure of the physicians can be analyzed and used as training input for automated controllers. Furthermore it is planned to observe various patients' reactions to certain drugs and use this information to improve the simulation environment [5], which is used to test the controllers. It is also possible, that the 
analysis of the data gives deeper insights into common critical situations, which occur frequently after a specific operation or precondition. The collected data will be used to improve the automated controllers in development in our projects in the cardiac intensive care unit. The system however is not limited to cardiac applications but can be used in various intensive care units. Furthermore there are plans to connect a small monitor and a keyboard to this hardware setup, so it can be used as a base for the final automated controlling device too.

\section{ACKNOWLEDGMENT}

This research was funded by the Graduate School of Information Science in Health (GSISH) and the Technical University of Munich (TUM) Graduate School.

\section{REFERENCES}

[1] N. Sprunk, A. Mendoza, A. Knoll, U. Schreiber, S. Eichhorn, J. Hörer and R. Bauernschmitt, "Hemodynamic regulation using fuzzy logic," in FSKD. IEEE, 2011, pp. 515-519.

[2] A. Mendoza G., N. Sprunk, B. Baumgartner, U. Schreiber, S. Eichhorn. R.Bauernschmitt, R. Lange, M. Krane, and A. Knoll, "Automation of an extracorporeal support system with adaptive fuzzy controllers," in Engineering in Medicine and Biology Society, EMBC, 2011 Annual International Conference of the IEEE, 2011, Sept. 3 2011, pp. 10331036.

[3] W. Haddad, J. Bailey, T. Hayakawa, and N. Hovakimyan, "Neural network adaptive output feedback control for intensive care unit sedation and intraoperative anesthesia," Neural Networks, IEEE Transactions on,vol. 18, no. 4, pp. $1049-1066$, july 2007.

[4] N. Padhye, S. Hamlin, A. Brazdeikis, and S. Hanneman, "Cardiovascular impact of manual and automated turns in icu," in Engineering in Medicine and Biology Society, 2009. EMBC 2009. Annual International Conference of the IEEE, Sept. 2009, pp. 1844 1847.

[5] N. Sprunk, A. Mendoza Garcia, U. Schreiber, R. Bauernschmitt, and A Knoll, "Cardiovascular model for development and test of automated hemodynamicregulation with medication," in Computing in Cardiology, 2011, Sept. 2011, pp. $153-156$ 